\title{
Polarization state and level repulsion in two-dimensional phononic crystals and waveguides in the presence of material anisotropy
}

\author{
Younes Achaoui, Abdelkrim Khelif, Sarah Benchabane, and \\ Vincent Laude \\ Institut FEMTO-ST, Université de Franche-Comté, CNRS, ENSMM, UTBM; 32 \\ avenue de l'Observatoire F-25044 Besançon, France
}

\begin{abstract}
We investigate the polarization of Bloch waves in two dimensional piezoelectric phononic crystals and phononic crystal waveguides managed therein. It is found that in addition to the strong coupling induced for waves polarized in the plane of the periodic structuration, a weaker but non negligible coupling of polarization components originates from material anisotropy. Numerical illustrations are given for an array of air holes in lithium niobate arranged according to a square lattice. It is observed that when a band mostly polarized in-plane gets close to a band mostly polarized out-of-plane, a phenomenon of repelling can occur, that in some instances introduces a local band gap. This interaction is accompanied by a transfer of the polarization state from one band to the other.
\end{abstract}

PACS numbers: 43.20.+g, 43.35.+d, 77.65.Dq 


\section{1. introduction}

Phononic crystals are periodic structures that can give rise to complete band gaps (BG) for acoustic waves in fluids or elastic waves (acoustic phonons) in solids [1, 2], in the very same way that photonic crystals prohibit the propagation of optical or electromagnetic waves $[3,4]$. The dispersion of the bands composing the band structure, the frequency position and the width of the band gaps are conditioned by the contrast between material constants of the constituent media on the one hand, and by the filling fraction, the geometrical shape of the inclusions and the lattice topology on the other hand. Within a frequency band gap, a phononic crystal acts as a mirror for incident waves, as a result of destructive interferences between waves scattered on the periodic inclusions. Thanks to the wide operating frequency range of acoustic and elastic waves, complete band gaps have been demonstrated theoretically and experimentally at different scales, for bulk waves $[2,5,6]$ and surface waves $[7,8,9,10,11]$, as well as for phononic crystal slabs $[12,13,14]$. Phononic crystals also allow for the obtaining of confined states or guided waves through the introduction of point or linear defects $[15,16,17,18]$. Phononic waveguides, resonators and stubs have been proposed as possible ways to create filtering and multiplexing structures based on the coupling of resonance and waveguiding phenomena [19, 20, 21].

In the case of acoustic waves in fluids, because of the single polarization involved (longitudinal), the band structure in the perfect crystal case and the transmission coefficient in the waveguide case are usually considered sufficient to characterize a phononic crystal. But for elastic waves propagating in a solid, both transverse and longitudinal polarizations exist and are possibly coupled owing to the periodic structuration. Taking the polarization state into account in the analysis of band diagrams is hence compulsory to show a complete picture of elastic wave propagation. This has for example been shown by a previous study dedicated to polarization effects in a perfect $2 \mathrm{D}$ phononic crystal made of air inclusions in an epoxy matrix [22]. This work highlighted the influence of the filling fraction on the coupling between in-plane transverse and longitudinal polarizations, where in-plane refers to the plane normal to the inclusion axis. The in-plane polarization components were found to be more coupled for higher filling fractions and a continuous variation of the polarization when the wavevector sweeps the first Brillouin zone was reported. This continuity of the elastic displacement fields along band structures has also been investigated in order to study the repulsion level between different branches in the band diagram for both one[23] or two- [24] dimensional phononic crystals. We are not aware of similar works for phononic crystals involving anisotropic materials or phononic waveguides, though the dependence of the guided waves dispersion on a change in the central inclusion radius or in the waveguide width has been reported recently [25, 26]. With the configurations proposed in these works, some branches initially located outside the band gap in the case of the perfect phononic crystal can enter or exit the band gap when the dimensions of the defect vary. The displacement field of some confined modes was reported as well 


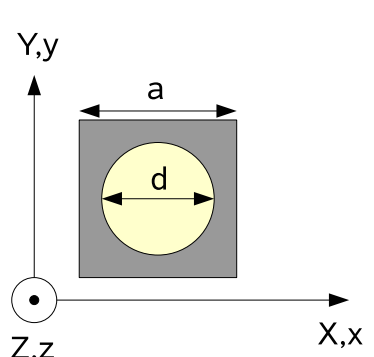

(a)

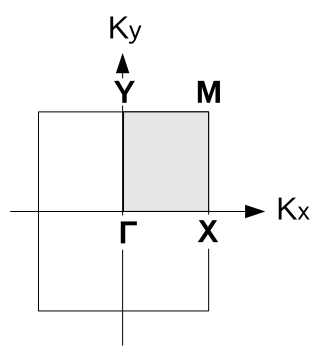

(b)

Figure 1. (a) 2D piezoelectric phononic crystal consisting of a two-dimensional squarelattice array of circular cylindrical holes in lithium niobate. (b) Corresponding first Brillouin zone.

and it was observed that following the same band as the Bloch wavevector varies in the first Brillouin zone, the polarization of the displacement field can change partially or totally. A detailed investigation of this phenomenon however remains to be performed.

In this paper, we first investigate the consequences of material anisotropy on the coupling of polarization components in two-dimensional phononic crystals. Of particular relevance is the combination of the effects of material anisotropy and of the periodic structuration. It is well known that a two-dimensional periodic structuration introduces a coupling for the in-plane polarization components, while the out-of-plane component remains decoupled. We here show that material anisotropy can result in the coupling of all polarization components. For illustration purposes, we numerically investigate the evolution of the polarization of Bloch waves in a two-dimensional piezoelectric phononic crystal composed of a square-lattice array of holes in lithium niobate $\left(\mathrm{LiNbO}_{3}\right)$. As a piezoelectric material, $\mathrm{LiNbO}_{3}$ is anisotropic for elastic wave propagation, so that in general the two shear and the longitudinal components of the polarization are not decoupled. The coupling induced by the anisotropy of $\mathrm{LiNbO}_{3}$ is rather weak compared to the coupling induced by the periodic structuration, but will be shown not to be negligible. Along our analysis, we first concentrate on the perfectly periodic phononic crystal. The plane wave expansion (PWE) method combined with an energy balance criterion are used to compute band structures displaying additional information related to the weighting of each polarization. We underline cases where polarizations are exchanged between interacting bands or conversely when this exchange does not happen upon band crossing. The study is then extended to the case of a phononic waveguide obtained by inserting a line defect in the initial phononic crystal thanks to the super-cell technique.

\section{Polarization coupling}

In this section, we investigate the influence of the anisotropy on the coupling of polarization components in phononic crystals. The considered geometry, and the definition of axes and Brillouin zone are given in Figure 1. The following analysis as well 
as the computation of band structures and modal distributions in the next sections are based on the PWE method. The PWE method is based on a direct application of the Bloch-Floquet theorem to the representation of Bloch waves as the product of a periodic function, given by a discrete sum over Fourier harmonics in the reciprocal-lattice space, with a time-harmonic exponential function with frequency $\omega$ and Bloch wave vector $\mathbf{k}$. For instance, the displacements in the $x$ direction read

$$
u_{x}(\mathbf{r})=\left(\sum_{n=1}^{N} U_{x n} \exp \left(-\imath \mathbf{G}^{n} \mathbf{r}\right) \exp (-\imath \mathbf{k} \cdot \mathbf{r})\right),
$$

where the $\mathbf{G}^{n}$ are the reciprocal lattice vectors and $\imath^{2}=-1$. Similar expressions hold for $u_{y}$ and $u_{z}$. We specifically use the formulation by Wilm et al. for bulk and plate waves in piezoelectric media [27], which was later extended to surface waves in anisotropic and piezoelectric media $[9,10]$. The representation of hollow inclusions follows the procedure exposed in Ref. [10]. The secular equation (Equation 5 of Ref. [10]),

$$
\omega^{2} \tilde{R} \tilde{U}=\left(\sum_{i, j=1,3} \Gamma_{i} \tilde{A}_{i j} \Gamma_{j}\right) \tilde{U}
$$

defines an eigenvalue problem for the frequency as a function of the wave vector and is used to obtain band structures. In this expression, $\tilde{U}$ is a vector gathering the Fourier coefficients of the three displacements and the electric potential, and the matrices $\Gamma_{i}$, $\tilde{A}_{i j}$, and $\tilde{R}$ contain $4 N \times 4 N$ Fourier coefficients. The detailed expressions of the different matrices will be useful to investigate the incidence of anisotropy on Bloch waves and their polarization. These matrices read

$$
\begin{aligned}
& \tilde{A}_{i j}=\left[\begin{array}{cccc}
A_{i j \mathbf{0}} & A_{i j \mathbf{G}^{1}-\mathbf{G}^{2}} & \ldots & A_{i j \mathbf{G}^{1}-\mathbf{G}^{N}} \\
A_{i j \mathbf{G}^{2}-\mathbf{G}^{1}} & A_{i j \mathbf{0}} & \ldots & A_{i j \mathbf{G}^{2}-\mathbf{G}^{N}} \\
\vdots & \vdots & \ddots & \vdots \\
A_{i j \mathbf{G}^{N}-\mathbf{G}^{1}} & A_{i j \mathbf{G}^{N}-\mathbf{G}^{2}} & \ldots & A_{i j \mathbf{0}}
\end{array}\right], \\
& \Gamma_{i}=\left[\begin{array}{cccc}
\left(k_{i}+G_{i}^{1}\right) I_{d} & & & 0 \\
& \left(k_{i}+G_{i}^{2}\right) I_{d} & & \\
& & \ddots & \\
0 & & & \left(k_{i}+G_{i}^{N}\right) I_{d}
\end{array}\right] \text {, } \\
& \tilde{R}=\left[\begin{array}{cccc}
\rho_{\mathbf{0}} \tilde{I} & \rho_{\mathbf{G}^{1}-\mathbf{G}^{2}} \tilde{I} & \ldots & \rho_{\mathbf{G}^{1}-\mathbf{G}^{N}} \tilde{I} \\
\rho_{\mathbf{G}^{2}-\mathbf{G}^{1}} \tilde{I} & \rho_{\mathbf{0}} \tilde{I} & \ldots & \rho_{\mathbf{G}^{2}-\mathbf{G}^{N}} \tilde{I} \\
\vdots & \vdots & \ddots & \vdots \\
\rho_{\mathbf{G}^{N}-\mathbf{G}^{1}} \tilde{I} & \rho_{\mathbf{G}^{N}-\mathbf{G}^{2}} \tilde{I} & \ldots & \rho_{\mathbf{0}} \tilde{I}
\end{array}\right]
\end{aligned}
$$

with $A_{i l \mathbf{G}}(j, k)=c_{i j k l \mathbf{G}}, A_{i l \mathbf{G}}(j, 4)=e_{l i j \mathbf{G}}, A_{i l \mathbf{G}}(4, k)=e_{i k l \mathbf{G}}, A_{i l \mathbf{G}}(4,4)=-\epsilon_{i l \mathbf{G}} \cdot I_{d}$ is the $4 \times 4$ identity matrix and $\tilde{I}=I_{d}$ but for $\tilde{I}(4,4)=0 . c_{i j k l}, e_{i j k}$, and $\epsilon_{i j}$ are the elastic, piezoelectric and dielectric tensors, respectively. The $\Gamma_{i}$ and $\tilde{R}$ matrices are each formed of $N^{2} 4 \times 4$ diagonal blocks. Material anisotropy enters the $\tilde{A}_{i j}$ matrices only, and the structure and symmetries of these matrices are directly dependent on those of 
the material tensors $c_{i j k l}, e_{i j k}$ and $\epsilon_{i j}$. In turn, the influence of anisotropy in the secular equation (2) is contained in the matrix

$$
\tilde{A}=\sum_{i, j=1,3} \Gamma_{i} \tilde{A}_{i j} \Gamma_{j}
$$

appearing in the right-hand side. $\tilde{A}$ retains the block structure of the $\tilde{A}_{i j}$ matrices. More precisely, the $(m, n)$-th sub-block reads

$$
\tilde{A}_{\mathbf{G}^{m}-\mathbf{G}^{n}}=\sum_{i, j=1,3}\left(k_{i}+G_{i}^{m}\right)\left(k_{j}+G_{j}^{n}\right) A_{i j \mathbf{G}^{m}-\mathbf{G}^{n}} .
$$

The above expressions can be further detailed for a two-dimensional phononic crystal. In this case, the summation $i, j=1,2$ and using the contracted notation for tensors we arrive at

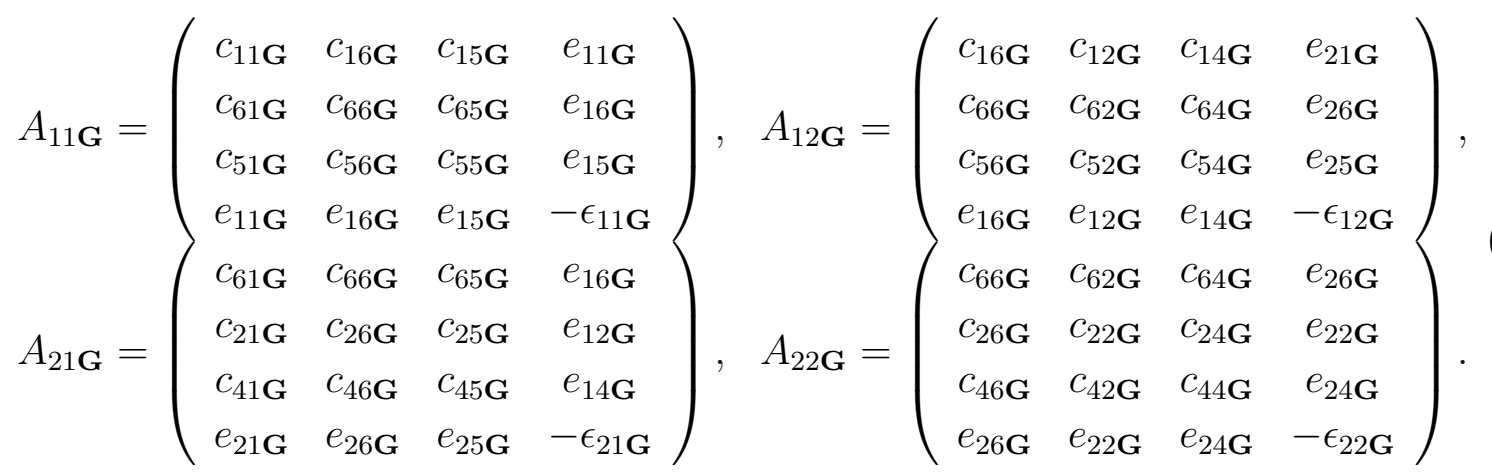

In the absence of any structuration, i.e for a homogeneous material, the summations on the reciprocal lattice vectors would be limited to $\mathbf{G}=0$, and the matrix $\tilde{A}$ would be formally equivalent to the Christoffel tensor [28]. In this case, propagation in the $x$ direction would only involve the $A_{110}$ matrix (resp. $A_{220}$ for propagation in the $y$ direction). The periodicity of the phononic crystal is manifested by non zero $\mathbf{G}^{m}$ and $\mathbf{G}^{n}$ components for $(m, n) \neq 0$ and causes a mixing of matrix elements in Equation (7), whatever the propagation direction $\mathbf{k}$.

In the case of general anisotropic media, inspection of the block matrices in Equation (8) reveals which polarization components are coupled. Such an analysis is straightforward but should be conducted for every crystallographic symmetry class and every material orientation considered. As an example, we have considered two cases in Table 1 . In the case of materials with isotropic or cubic crystalline symmetry (e.g., silicon), the periodic structuration results in non-zero off-diagonal terms coupling displacements $u_{x}$ and $u_{y}$, but the out-of-plane displacement $u_{z}$ and the electric potential $\phi$ are uncoupled. This directly explains the coupling between in-plane polarization components and the decoupling of the out-of-plane polarization component in isotropic $2 \mathrm{D}$ phononic crystals. The case of materials with trigonal $3 m$ symmetry (e.g., lithium niobate) is different. In the homogeneous material, propagation along the $x$ and $y$ directions occurs according either to a non-piezoelectrically coupled wave (decoupled $u_{x}$ polarization), or to piezoelectrically coupled waves (coupled $u_{y}, u_{z}$, and $\phi$ polarization). With the additional consideration of a periodic structuration in the $(x, y)$ plane, all polarization components get coupled. This analysis, of course, is based only on the 
Table 1. Shapes of the $A_{i j}$ matrices in the case of materials with isotropic, cubic, and trigonal $3 m$ crystalline symmetry. Orientation along the principal crystallographic axes is assumed. Zero elements are indicated by dots (.) while non-zero values are indicated by circled crosses $(\otimes)$.

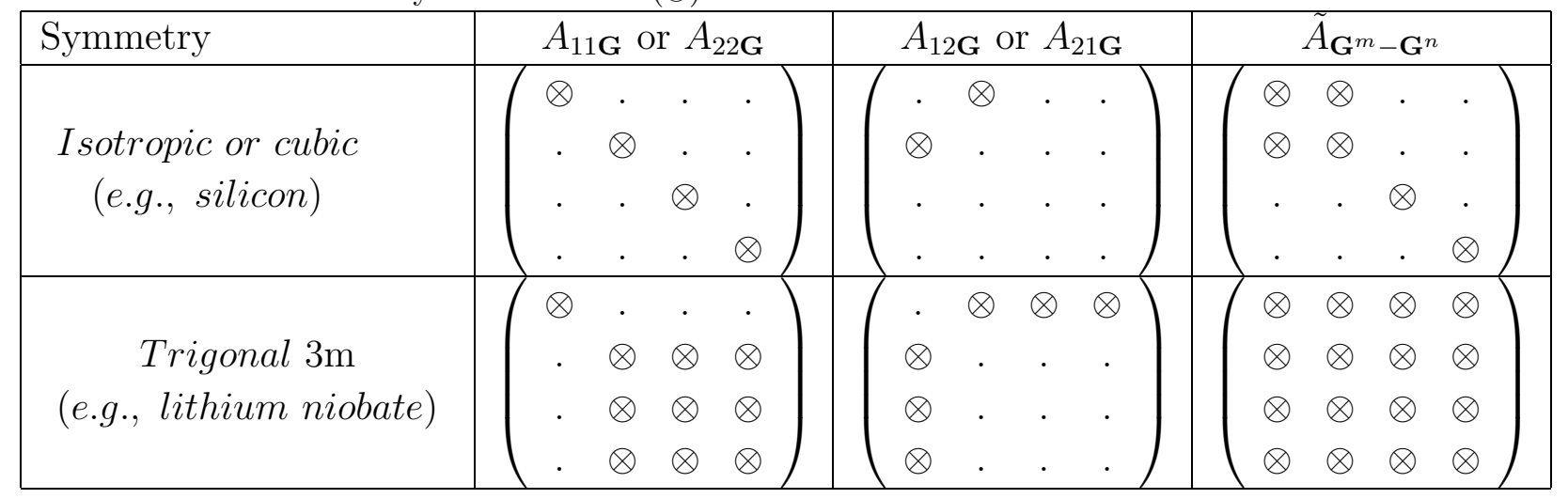

nullity or not of matrix elements, and the strength of the coupling induced by the periodic structuration and material anisotropy depends quantitatively on the actual magnitude of the matrix elements. Numerical illustrations are given in the subsequent Sections in the case of lithium niobate.

\section{Two-dimensional piezoelectric phononic crystal}

We consider in the following a two-dimensional piezoelectric phononic crystal made of a square-lattice array of circular cylindrical holes in $\mathrm{LiNbO}_{3}$. The crystallographic orientation of $\mathrm{LiNbO}_{3}$ is chosen to be the Z-cut, so that the $\mathrm{Z}$ crystallographic axis is parallel to the $z$ axis of the reference frame of Figure 1a. The filling fraction is $64 \%$. Theoretical and experimental properties of such a phononic crystal have been investigated before, and the existence of a complete band gap for both bulk and surface waves has been demonstrated $[10,11,29]$. Here we further consider the evolution of the polarization of Bloch waves.

The two dimensional Fourier expansions are truncated to the 36 first harmonics in this section, as a result of a compromise between convergence and computation time, but also to obtain band structures that are directly comparable with those obtained for waveguides with the super-cell technique in the next section. With this choice, convergence in terms of frequencies is within a few per cent of the high number of harmonics limit.

In order to avoid possible confusions, we use throughout this paper the displacement field components $u_{x}, u_{y}$, and $u_{z}$ to represent polarizations. In this way, we avoid using the terminology of longitudinal, horizontal and vertical shear polarizations, which are dependent on the direction of propagation. In the band structures, polarization is represented by three positive numbers summing to unity. For instance, the amount of 

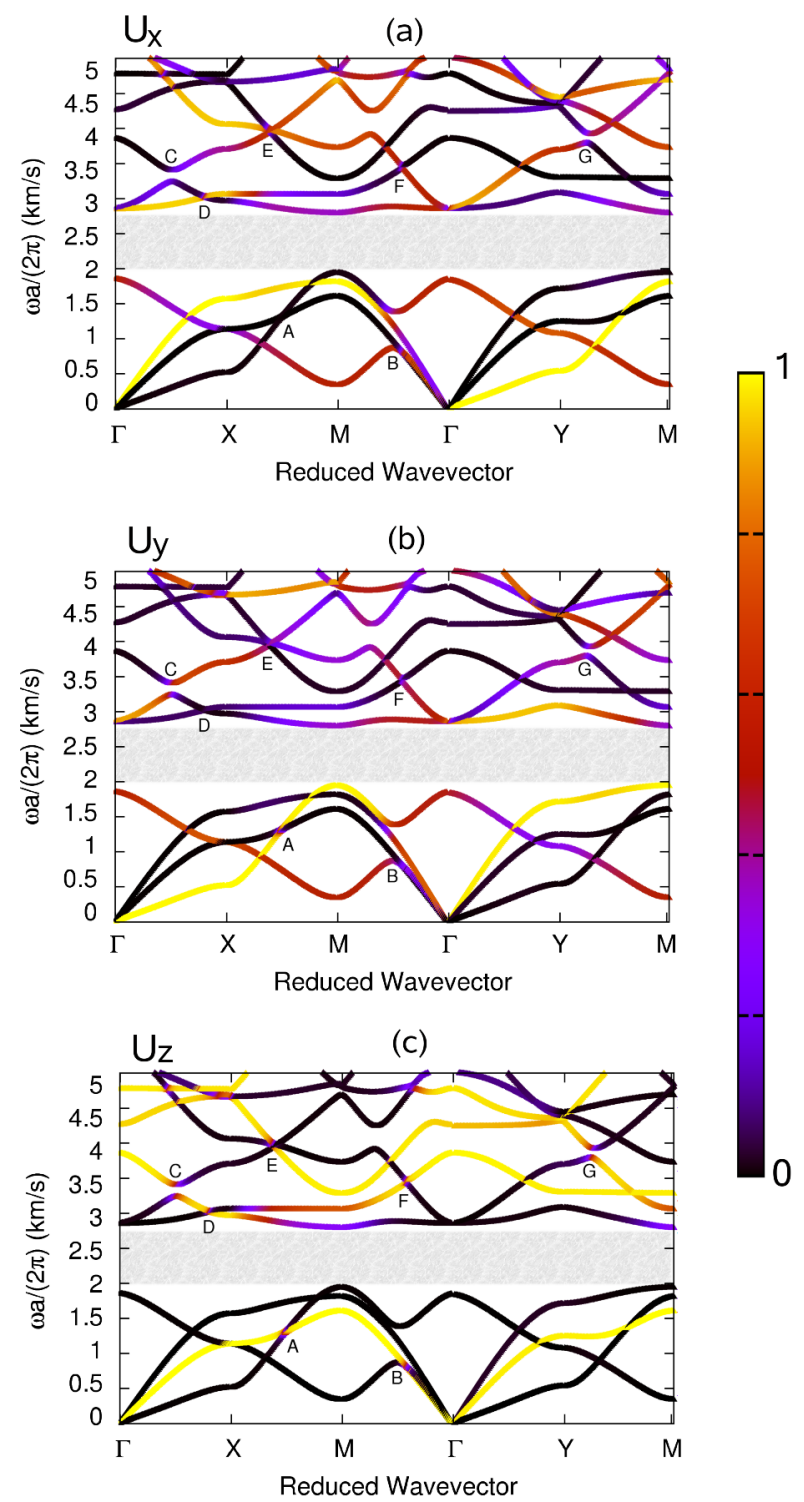

Figure 2. Band structure of the phononic crystal depicted in Figure 1a. The complete band gap is indicated by the grayed region. The three band structures depict the same dispersion relations but the coloring of the bands shows the amount of polarization along (a) the $x$ axis, (b) the $y$ axis and (c) the $z$ axis. Points A, B, C, D, E, F and G mark the $(k, \omega)$ positions of the first seven intersections of repelling branches.

polarization along the $x$ direction is given by

$$
p_{x}^{2}=\frac{\int\left|u_{x}\right|^{2} d \mathbf{r}}{\int\left(\left|u_{x}\right|^{2}+\left|u_{y}\right|^{2}+\left|u_{z}\right|^{2}\right) d \mathbf{r}},
$$

with the integral taken over the unit-cell. Similar expressions hold for the amounts of polarization $p_{y}^{2}$ and $p_{z}^{2}$ measured along the $y$ and the $z$ axes, respectively.

Figure 2 displays the band structure giving the dispersion relation for elastic waves propagating in the phononic crystal of Figure 1a. The structure shows a complete band gap with a $33 \%$ fractional bandwidth. The three band structures actually display the 

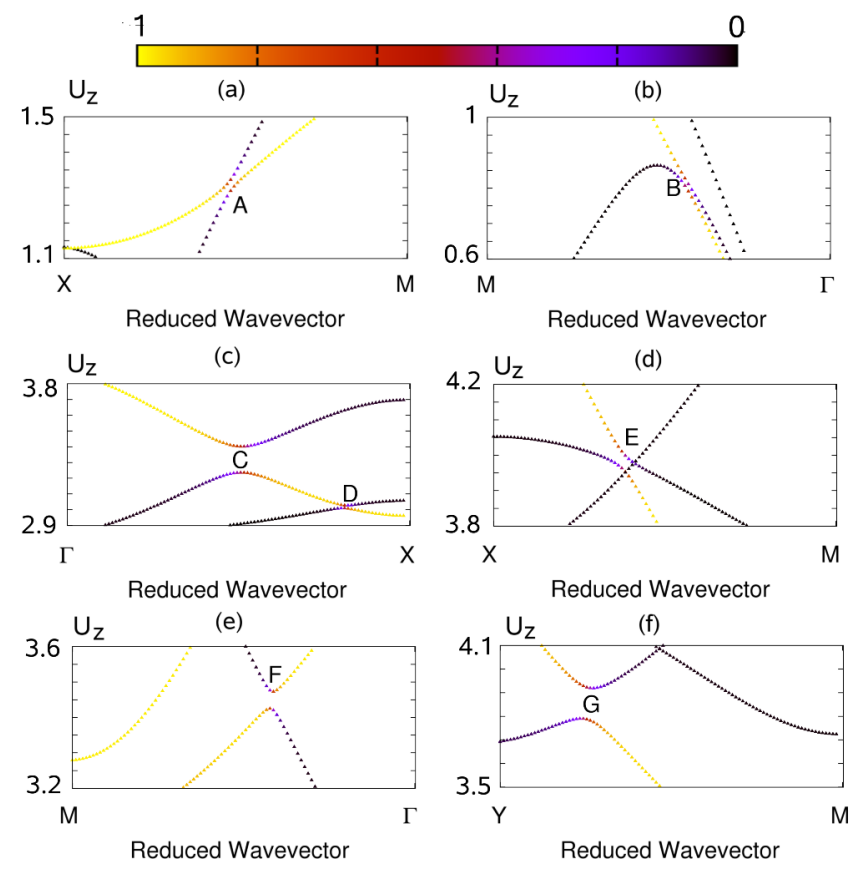

Figure 3. Enlarged dispersion relations in Figure 2c in the vicinity of repelling bands near (a) point $\mathrm{A}$, (b) point $\mathrm{B}$, (c) point $\mathrm{C}$ and $\mathrm{D}$, (d) point $\mathrm{E}$, (e) point $\mathrm{F}$ and (f) point G.

same $\omega(k)$ dispersion relations, but with the additional information of the amounts of polarization $p_{x}^{2}, p_{y}^{2}$, and $p_{z}^{2}$ in Fig. 2a, Fig. $2 \mathrm{~b}$ and Fig. 2c, respectively.

It can be observed in Figure 2 that the bands do not in general possess a pure polarization, except for some portions of them. The coupling of the in-plane polarization components $\left(u_{x}\right.$ and $\left.u_{y}\right)$, however, appears much stronger than the coupling with the outof-plane component $\left(u_{z}\right)$, especially above the complete band gap. This observation is fully consistent with the results of the previous Section. If it were not for the anisotropy of $\mathrm{LiNbO}_{3}$, the two-dimensional periodic structuration would introduce a strong coupling of the in-plane polarization components, but the out-of-plane component would remain completely decoupled. The coupling between in-plane polarization components is especially strong in Fig. 2 for propagation directions that encompass the M point of the first Brillouin zone. The comparatively weaker coupling of all three polarization components is caused by material anisotropy.

As a general rule, it can be observed that the polarization varies continuously as the Bloch wave vector sweeps the first Brillouin zone. There are, however, intriguing points in the band structure where bands cross without interacting or conversely interact and repel each other. Points at which repelling between bands occurs have been labeled A to $\mathrm{G}$ in Figure 2. Figure 3 displays closer views at these seven points, with the color indicating the $p_{z}^{2}$ component of the polarization. Each couple of repelling bands is composed of a mostly in-plane polarized band and of a mostly out-of-plane polarized band, which leads us to the conclusion that repelling occurs as a result of the coupling provided by the anisotropy of $\mathrm{LiNbO}_{3}$. It can be observed that when bands repel, they 


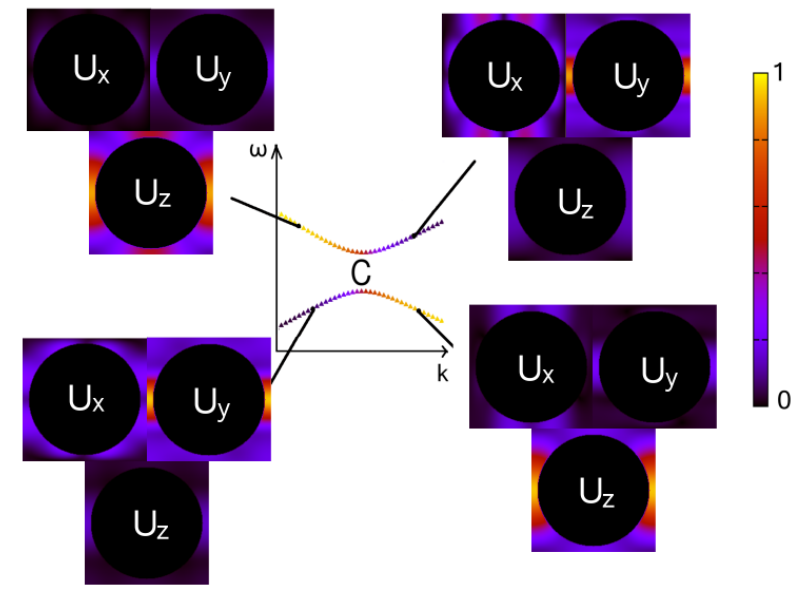

Figure 4. Spatial distribution of the polarization components $u_{x}, u_{y}$ and $u_{z}$ of the displacement field for points before and after the repulsion in point $\mathrm{C}$.

exchange their polarization state, so that the polarization remains a continuous function of the wave vector $\mathbf{k}$.

Two different cases are further observed. Repelling at points C, D, F and G introduces a local band gap, while, conversely, repelling at points A, B and E does not introduce any. We observe that the occurrence of a local band gap is conditioned by the two repelling bands being either on opposite sides of a horizontal line passing at the repelling point (presence of a local BG), or on the same side (absence of a local $B G)$.

Figure 4 illustrates in more detail how the polarization is transferred between the two repelling bands at point $\mathrm{C}$. At the left of point $\mathrm{C}$, the upper band is mostly polarized along $z$, while the lower band is mostly polarized in-plane and rather along the $y$ axis. After repelling, the respective polarizations have been exchanged from out-of-plane to in-plane, and vice versa.

\section{Piezoelectric phononic crystal waveguide}

In this Section, we consider a phononic crystal waveguide managed by removing a row of holes from the phononic crystal along the $x$ direction and investigate how the polarization and repelling properties obtained in the previous section for the perfectly periodic phononic crystal are modified. The PWE method can be used to obtain the dispersion of guided waves for frequencies that fall within the complete band gap, using the super-cell technique $[25,26]$. In practice, the considered unit-cell shown in Figure 5 is made 7 times longer along the $y$ direction, as compared to the unit-cell in Figure 2, and thus includes three holes on both sides of the central guiding section. The holes have the same filling fraction as in the previous section and the number of Fourier harmonics has been increased by a factor of 6 in the $y$ direction to achieve convergence conditions similar to those obtained in the previous section. Because of periodicity in the computations, 


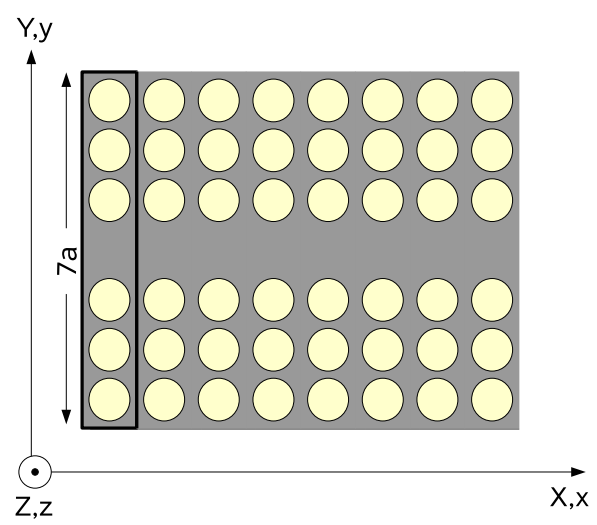

Figure 5. Phononic crystal waveguide created by removing a row of holes from the phononic crystal along the $x$ direction. The framed box shows the unit-cell used for super-cell computations.

six holes separate neighboring waveguides. We have checked that this separation is actually sufficient to isolate the periodically repeated waveguides by computing the band structure along the $\Gamma Y$ direction of the first Brillouin zone and verifying that only flat bands are obtained within the complete band gap (if we had obtained dispersion then this would have been an indication of coupling between adjacent waveguides).

Figure 6 displays the dispersion relations of Bloch waves guided by the structure in Figure 5 and propagating along the $x$ direction for frequencies within the complete phononic band gap. As for the band structure of the perfectly periodic phononic crystal depicted in Figure 2, the band structure for guided Bloch waves is repeated three times with the additional information of the amounts of polarization $p_{x}^{2}, p_{y}^{2}$, and $p_{z}^{2}$ in Figures 6a, 6b, and 6c, respectively. Eight different bands are apparent and are numbered sequentially. Bands 1 and 4 are mostly polarized in-plane and repel midways between the $\Gamma$ and the X points. Bands 2 and 6 are also mostly polarized in-plane. For all these bands, the distribution between in-plane components $p_{x}$ and $p_{y}$ does not remain constant as the wavevector varies from the $\Gamma$ to the $\mathrm{X}$ points. Band 3 is purely polarized out-of-plane (vertical shear wave), while band 8 is mostly polarized out-ofplane. The cases of bands 5 and 7 are more intriguing, since these two bands are respectively mostly polarized in-plane and out-of-plane before point I and J where they repel, create a local band gap, and abruptly exchange their polarization state. The mechanism for the coupling between these two bands is made possible thanks to the anisotropy of $\mathrm{LiNbO}_{3}$, similarly to what was discussed in Section II.

Figure 7 illustrates how polarization transfer occurs at the repelling of bands 5 and 7. We have chosen to show the modulus of the real part of the displacements $u_{x}$. It can be observed that the distribution of $u_{x}$ at point $\mathrm{K}$ on band 7 is transferred without almost any alteration to point $\mathrm{J}$ on band 5 . At the wavevector for which the bands are the closest, i.e. at points I and L, the modal distributions are very similar. In the absence of anisotropy, the repelling point would have been replaced by a crossing of the two bands, and points $\mathrm{H}$ and $\mathrm{M}$ would have been on the same band (similarly, points $\mathrm{J}$ 

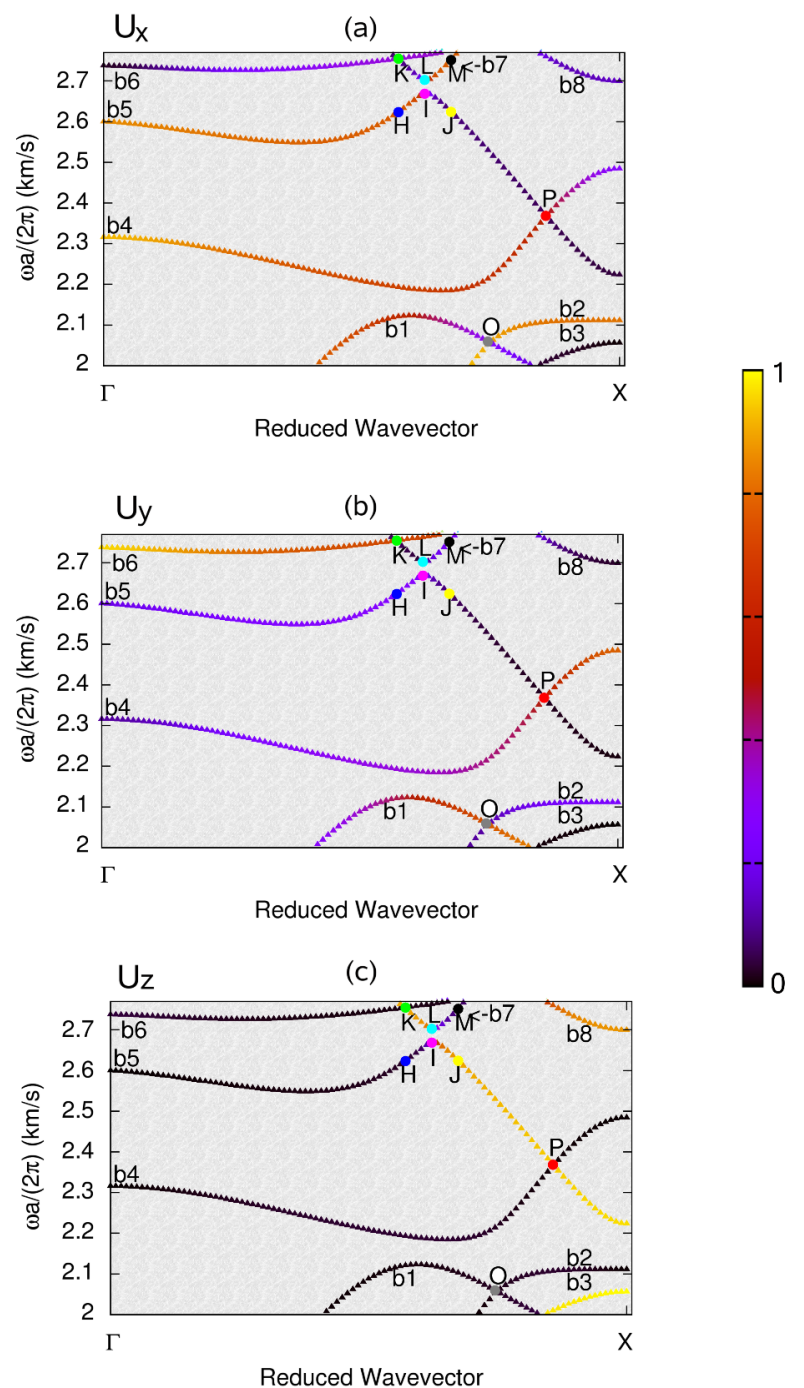

Figure 6. Band structure along the $\Gamma \mathrm{X}$ direction for the phononic waveguide displayed in Figure 5, showing the dispersion relation for guided Bloch waves. The band structure is repeated three times with the information of the amount of polarization (a) $p_{x}^{2}$, (b) $p_{y}^{2}$, and (c) $p_{z}^{2}$.

and $\mathrm{K}$ would have been on the same band).

\section{Conclusion}

In summary, we have investigated the polarization of Bloch waves in a two dimensional piezoelectric phononic crystal and a phononic crystal waveguide managed inside it. By examining the structure of the matrices involved in the secular equation and by studying band structures, it was found that in addition to the strong coupling induced for waves polarized in the plane of the periodic structuration, a weaker but non negligible coupling of all polarization components originates from material anisotropy. As a consequence, when a band mostly polarized in-plane gets close to a band mostly polarized out- 

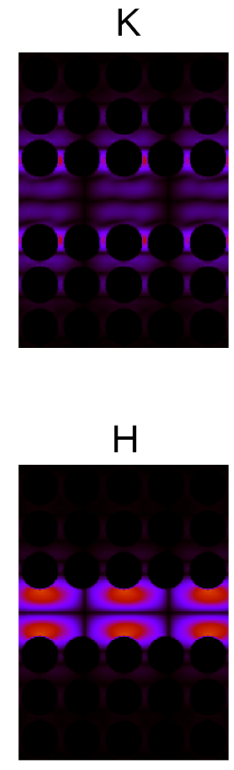

M

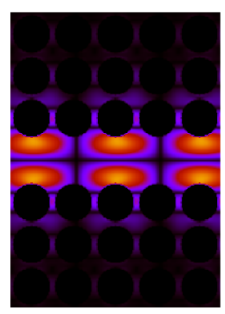

$J$

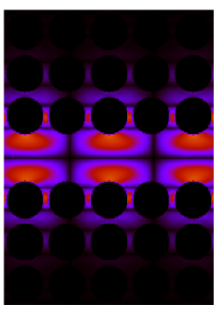

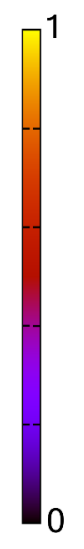

Figure 7. Spatial distribution of the modulus of the real part of the displacements $u_{x}$ at the repelling of bands 5 and 7 , shown for the six points $\mathrm{H}$ to $\mathrm{M}$ indicated in Figure 6. Points H, I, and $\mathrm{J}$ are placed along band 5 before, at and after the repelling point, respectively. Points K, L and M are similarly placed along band 7 .

of-plane, a phenomenon of repelling can occur between them that in some instances introduces a local band gap. This interaction is accompanied by a transfer of the polarization state from one band to the other. The findings in this paper illustrate that when anisotropic materials are involved, dispersion relations for Bloch waves in phononic crystals in the form $\omega(k)$ do not give a complete picture of wave propagation and must be supplemented with the dependence of the polarization on the wavevector.

[1] M. Sigalas and E. N. Economou. Band structure of elastic waves in two dimensional systems. Solid State Comm., 86:141, 1993.

[2] M. S. Kushwaha, P. Halevi, L. Dobrzynski, and B. Djafari-Rouhani. Acoustic band structure of periodic elastic composites. Phys. Rev. Lett., 71:2022, September 1993.

[3] E. Yablonovitch. Inhibited spontaneous emission in solid-state physics and electronics. Phys. Rev. Lett., 58:2059, May 1987.

[4] S. John. Strong localization of photons in certain disordered dielectric superlattices. Phys. Rev. Lett., 58:2486, June 1987.

[5] M. S. Kushwaha, P. Halevi, G. Martinez, L. Dobrzynski, and B. Djafari-Rouhani. Theory of acoustic band structure of periodic elastic composites. Phys. Rev. B, 49:2313, 1994.

[6] M. S. Kushwaha and P. Halevi. Band-gap engineering in periodic elastic composites. Appl. Phys. Lett., 64:1085, February 1994.

[7] Y. Tanaka and S. I. Tamura. Surface acoustic waves in two-dimensional periodic elastic structures. Phys. Rev. B, 58:7958, 1998.

[8] Y. Tanaka and S. I. Tamura. Acoustic stop bands of surface and bulk modes in two-dimensional phononic lattices consisting of aluminum and a polymer. Phys. Rev. B, 60:13294, 1999.

[9] T.-T Wu, Z. G. Huang, and S. Lin. Surface and bulk acoustic waves in two-dimensional phononic crystal consisting of materials with general anisotropy. Phys. Rev. B, 69:094301, 2004.

[10] V. Laude, M. Wilm, S. Benchabane, and A. Khelif. Full band gap for surface acoustic waves in a piezoelectric phononic crystal. Phys. Rev. E, 71:036607, 2005. 
[11] S. Benchabane, A. Khelif, J.-Y. Rauch, L. Robert, and V. Laude. Evidence for complete surface wave band gap in a piezoelectric phononic crystal. Phys. Rev. E, 73:065601(R), June 2006.

[12] A. Khelif, B. Aoubiza, S. Mohammadi, A. Adibi, and V. Laude. Complete band gaps in twodimensional phononic crystal slabs. Phys. Rev. E, 74:046610, 2006.

[13] J. C. Hsu and T.-T. Wu. Propagation of surface acoustic waves through sharply bent twodimensional phononic crystal waveguides using a finit difference time domaine method. Phys. Rev. B, 74:144303, 2006.

[14] C. Charles, B. Bonello, and F. Ganot. Propagation of guided elastic waves in 2D phononic crystals. Ultrasonics, 44:1209(E), 2006.

[15] M. M. Sigalas. Defect states of acoustic waves in a two-dimensional lattice of solid cylinders. $J$. Appl. Phys., 84:3026, 1998.

[16] M. Torres, F. R. Montero de Espinosa, D. García-Pablos, and N. García. Sonic band gaps in finite elastic media: Surface states and localization phenomena in linear and point defects. Phys. Rev. Lett., 82:3054, April 1999.

[17] M. Kafesaki, M. M. Sigalas, and N. García. Frequency Modulation in the Transmittivity of Wave Guides in Elastic-Wave Band-Gap Materials. Phys. Rev. Lett., 85:4044, November 2000.

[18] H. Chandra, P. A. Deymier, and J. O. Vasseur. Elastic wave propagation along waveguides in three-dimensional phononic crystals. Phys. Rev. B, 70:054302, August 2004.

[19] A. Khelif, A. Choujaa, B. Djafari-Rouhani, M. Wilm, S. Ballandras, and V. Laude. Trapping and guiding of acoustic waves by defect modes in a full-band-gap ultrasonic crystal. Phys. Rev. B, 68:214301, December 2003.

[20] Y. Pennec, B. Djafari-Rouhani, J. O. Vasseur, A. Larabi, H. Khelif, A. Choujaa, S. Benchabane, and V. Laude. Acoustic channel drop tunneling in a phononic crystal. Appl. Phys. Lett., $87: 261912,2005$.

[21] J. H. Sun and T.-T. Wu. Analyses of mode coupling in joined parallel phononic crystal waveguides. Phys. Rev. B, 71:174303, 2005.

[22] B. Manzanares-Martinez and .F Ramos-Mendieta. Sagittal acoustic waves in phononic crystals: k-dependent polarization. Phys. Rev. B, 76:134303, 2007.

[23] H. Kato, H. J. Maris, and S. I. Tamura. Resonant-mode conversion and transmission of phonons in superlattices. Phys. Rev. B, 53:7884, 1996.

[24] T-T. Wu and Z-G. Huang. Level repulsions of bulk acoustic waves in composite materials. Phys. Rev. B, 70:214304, 2004.

[25] J. H. Sun and T. T. Wu. Propagation of acoustic waves in phononic crystal plates and waveguides using a finit difference time domain method. Phys. Rev. B, 76:104304, 2007.

[26] J. O. Vasseur, P.A. Deymier, B. Djafari-Rouhani, Y. Pennec, and A-C. Hladky-Hennion. Absolute forbiden bands and waveguiding in two-dimensional phononic crystal plates. Phys. Rev. B, 77:085415, 2008.

[27] M. Wilm, S. Ballandras, V. Laude, and T. Pastureaud. A full 3D plane wave expansion model for 1-3 piezoelectric composite structures. J. Acous. Soc. Am., 112:943, 2002.

[28] D. Royer and E. Dieulesaint. Ondes élastiques dans les solides, Vol. 1. Masson, Paris, 1999.

[29] K. Kokkonen, M. Kaivola, S. Benchabane, A. Khelif, and V. Laude. Scattering of surface acoustic waves by a phononic crystal revealed by heterodyne interferometry. Appl. Phys. Lett., 91:083517, 2007. 